\title{
On the generation of asymmetric warps in disk galaxies
}

\author{
K. Saha and C. J. Jog
}

Department of Physics, Indian Institute of Science, Bangalore 560012, India

e-mail: [kanak;cjjog]@physics.iisc.ernet.in

Received 23 July 2005 / Accepted 30 September 2005

\section{ABSTRACT}

Context. The warps in many spiral galaxies are now known to be asymmetric. Recent sensitive observations have revealed that asymmetry of warps may be the norm rather than exception. However there exists no generic mechanism to generate these asymmetries in warps.

Aims. To provide an explanation for the generation of asymmetric warps in disk galaxies

Methods. We have derived the dispersion relation in a compact form for the S-shaped warps (described by the $m=1$ mode) and the bowlshaped distribution (described by the $m=0$ mode) in a galactic disk embedded in a dark matter halo. We then performed the numerical modal analysis and used the linear and time-dependent superposition principle to generate asymmetric warps in the disk.

Results. On doing the modal analysis we find the frequency of the $m=0$ mode is much larger than that of the $m=1$ mode. The linear and time-dependent superposition of these modes with their unmodulated amplitudes (that is, the coefficients of superposition being unity) results in an asymmetry in warps of $\sim 20-40 \%$, whereas a smaller coefficient for the $m=0$ mode results in a smaller asymmetry. The resulting values agree well with the recent observations. We study the dependence of the asymmetry index on the dark matter halo parameters. This approach can also naturally produce U-shaped warps and L-shaped warps.

Conclusions. We show that a rich variety of possible asymmetries in the $z$-distribution of the spiral galaxies can naturally arise due to a dynamical wave interference between the first two bending modes (i.e. $m=0$ and $m=1$ ) in the disk. This is a simple but general method for generating asymmetric warps that is independent of how the individual modes arise in the disk.

Key words. galaxies: kinematics and dynamics - galaxies: spiral - galaxies: structure

\section{Introduction}

The disks in most spiral galaxies are not flat and their outer parts often show warping of the material away from the galactic midplane. Warps are mostly seen in the atomic hydrogen gas (HI) in the outer parts of a galactic disk (Sancisi 1976; Bosma 1978), but there is also a strong observational evidence for optical warps in old stars, as in NGC 4565 (van der Kruit \& Searle 1981). A statistical analysis of a large number of edge-on spirals has shown that optical warps are seen in a significant fraction (about 40\%) of the spiral galaxy disks (Sanchez-Saavedra et al. 1990; Reshetnikov \& Combes 1998, 1999).

Thus the warps are a common phenomenon and hence must be either long-lived or be excited repeatedly. Tidal interactions have been proposed as a triggering mechanism for warps, however since many isolated spirals- such as NGC 4565 also show warps (Sancisi 1976), this cannot be the main triggering mechanism for warps. Anisotropic pressure applied by the intergalactic magnetic field can generate an S-shaped warp in the galactic disk (Battaner et al. 1990). But a very high value of the magnetic field is required to produce warp near 4-6 disk scalelengths. Alternatively gas accretion could generate warps repeatedly, as for example seen in the cosmological simulations of disk formation (Semelin \& Combes 2005).
One plausible mechanism to get long-lived warps is to use the modal approach first proposed and studied by Hunter \& Toomre (1969). Sparke \& Casertano (1988) (hereafter, SC88) followed Hunter \& Toomre's (1969) approach but also included a dark matter halo and achieved the above goal of avoiding differential precession. They have shown that in the presence of an oblate halo potential a system of concentric circular rings, representing the galactic disk, is able to accomplish a configuration where all the rings are synchronized to precess with a uniform precession frequency. Such modes are called normal modes, and in this case since the halo is included, it is a modified-tilt mode of the disk because it is derived by modifying the trivial tilt mode solution of the linearized equations of motion of an isolated system of rings.

The warps were initially deduced to have an S-shaped distribution, with equal amplitudes on both sides of the mid-plane. This makes their theoretical analysis easier. It has long been known, however, that quite a few galaxies show asymmetric warps so that the amplitudes on the two sides are appreciably different. For example, our own Galaxy has an asymmetric warp: the mean plane bends up to $4 \mathrm{kpc}$ above the plane of the inner Galaxy on the northern side of the disk, while it reaches down only half on the southern side and then turns back up toward the inner disk plane (see Burton 1988). More 
recent, sensitive observations have revealed that asymmetry of warps may be the norm rather than the exception as shown by the HI gas and optical $R$-band observations (Garcia-Ruiz et al. 2002). A detailed catalog of the measure of asymmetries in optical warps seen in edge-on galaxies can be found in Sanchez-Saavedra et al. (2003). Theoretically, S-shaped as well as asymmetric warps have been shown to arise via bending instabilities in the $N$-body studies of Revaz \& Pfenniger (2004); also these have been proposed to arise due to gas infall (Lopez-Corredoira et al. 2002).

In this paper we propose and study the generation of the asymmetric warps in disk galaxies. We propose that an asymmetric warp arises due to a dynamical wave interference between the first two stable bending modes (i.e. $m=0$ and $m=1$ ) in a gravitating system. We do not deal with the orign of these modes, we assume that some excitation mechanism - perhaps an external perturber, extragalactic magnetic field, or gas accretion, or internal bending instabilities - gives rise to both these modes and hence we do not include explicitly any source term in our analysis. It is reasonable that, once excited, these modes will interact with each other. We study a general case so that the modes need not even be triggered in a commensurate fashion. A different epoch of onset for the two modes will merely be reflected in a different initial ratio of the amplitudes of the modes.

It was demonstrated numerically by SC88 and Sparke (1995) respectively that warping mode $(m=1)$ and the bowlshaped mode $(m=0)$ are stable in a cold, thin self-gravitatiing axisymmetric disk. The basic idea of the present paper is to use this fact and then let these modes interfere with each other, and see if this results in an asymmetric warp. This idea of superposition of modes to explain the origin of asymmetric warps was first proposed by Sparke (1995), and also more recently by Lopez-Corredoira et al (2002) and Castro-Rodriguez et al. (2002), but they did not work out further details.

We do not address here the other important aspect of the warping phenomenon, namely its maintenance, which is a long-standing problem. In the modal approach some reasons for the reduction of warp lifetime are: back-reaction of the uniformly precessing disk onto a live dark matter halo (Nelson \& Tremaine 1995), and disk thickness and random motion (Sellwood 1996), which can damp out the disk warping in a few orbital time scales. Some numerical simulation studies (Dubinsky \& Kuijken 1995; Binney et al. 1998) have confirmed that the dynamical friction due to the oblate dark matter halo damps out a symmetric warp. On the other hand, the maintenance problem does not arise if there is gas accretion of $\sim$ few $M_{\odot} \mathrm{yr}^{-1}$ repeatedly generating warps as discussed above.

In this paper, we have isolated and studied one well-defined physical aspect of the problem, namely that if the $m=1$ and $m=0$ modes are excited due to any of the mechanisms mentioned above- namely, tidal interaction, or gas accretion, or bending instabilities, then it is inevitable that these will get superposed and we study the resulting behavior. Further, even if these modes are short-lived, if these can be excited repeatedly, then we can explain why a large fraction of spiral galaxies show asymmetric warps.
In Sect. 2, we formulate the equations for $m=0$ and $m=1$ cases, and give the numerical scheme for solving these, and also specify the input parameters for the disk and the halo. The results are described in Sect. 3, and Sect. 4 summarizes the conclusions from this paper.

\section{Formulation}

\subsection{Dynamics of bending modes}

We consider various possible bending modes in a cold, selfgravitating, thin axisymmetric disk in the presence of a rigid or non-responsive dark matter halo. The disk, with a radial surface density profile $\Sigma(r)$, rotates in the equatorial plane $(z=0)$ of the spheroidal dark matter halo with angular speed $\Omega(r)$ about the halo's symmetry axis $(r=0)$. Here $(r, \varphi, z)$ are the circular cylindrical polar coordinates. The dynamical system described above is the same as in SC88 and most of the formulation follows SC88.

The dynamical equation of a small bending of the disk perpendicular to its unperturbed plane $(z=0)$ is given by

$$
\left(\frac{\partial}{\partial t}+\Omega(r) \frac{\partial}{\partial \varphi}\right)^{2} \mathcal{Z}=\mathcal{F}_{\text {self }}+\mathcal{F}_{\text {halo }}
$$

where the small bending is described by a single function $\mathcal{Z}(r, \varphi, t)$ and $\mathcal{F}_{\text {self }}$ is the vertical force due to the bent disk itself. $\mathcal{F}_{\text {halo }}$ is the vertical restoring force near the disk plane $(z=0)$ due to the dark matter halo.

We consider the small bending of the disk normal to its plane as resulting from the linear superposition of different bending modes each described by an azimuthal wave number $m$. So the linear superposition allows us to write

$\mathcal{Z}(r, \varphi, t)=\sum_{m} A_{m} z_{m}(r, \varphi, t)$.

In the above relation $A_{m}$ is a constant number which denotes the coefficients of superposition and

$z_{m}(r, \varphi, t)=\mathfrak{R}\left\{h_{m}(r) \mathrm{e}^{\mathrm{i}\left(\omega_{m} t-m \varphi\right)}\right\}$

describes the bending mode of the disk with azimuthal wave number $m$, where $h_{m}(r)$ is the unmodulated amplitude of the $m$ th mode. Thus, the total amplitude of the $m$ th mode in the superposition is given by $A_{m} h_{m}(r)$.

The general dynamical equation for the bending mode with the azimuthal wavenumber $m$ is then obtained by substituting the form given by Eq. (2b) into Eq. (1):

$$
\begin{aligned}
& {\left[\left(\omega_{m}-m \Omega(r)\right)^{2}-v_{h}^{2}(r)\right] h_{m}(r)=} \\
& G \int_{0}^{\infty} \Sigma\left(r^{\prime}\right) H\left(r, r^{\prime}\right)\left[h_{m}(r)-h_{m}\left(r^{\prime}\right)\right] r^{\prime} \mathrm{d} r^{\prime} \\
& +G \int_{0}^{\infty} \Sigma\left(r^{\prime}\right) K_{m}\left(r, r^{\prime}\right) h_{m}\left(r^{\prime}\right) r^{\prime} \mathrm{d} r^{\prime}
\end{aligned}
$$

The angular speed $\Omega(r)$ appearing in above equation gets a contribution from the warped disk as well as from the dark matter halo described in Sect. 2.3. The definitions of $H\left(r, r^{\prime}\right)$ and $K_{m}\left(r, r^{\prime}\right)$ are as follows:

$$
H\left(r, r^{\prime}\right)=\int_{0}^{\infty} \frac{1}{\left[r^{2}+r^{\prime 2}-2 r r^{\prime} \cos \psi+z_{0}^{2}\right]^{\frac{3}{2}}} \mathrm{~d} \psi
$$


$K_{m}\left(r, r^{\prime}\right)=\int_{0}^{\infty} \frac{1-\cos (m \psi)}{\left[r^{2}+r^{\prime 2}-2 r r^{\prime} \cos \psi+z_{0}^{2}\right]^{\frac{3}{2}}} \mathrm{~d} \psi$.

These functions can be further expressed in terms of complete elliptical integrals. Note that for the bowl-shaped mode $(m=$ 0 ), the function $K_{0}\left(r, r^{\prime}\right)$ vanishes.

Equation (3a) is obtained by substituting in Eq. (1) the following:

$\mathcal{F}_{\text {halo }}^{m}(r, \varphi, t)=-v_{h}^{2}(r) z_{m}(r, \varphi, t)$

where $v_{h}$ is the vertical frequency due to the dark matter halo in the unperturbed disk plane.

$$
\begin{aligned}
& \mathcal{F}_{\text {self }}^{m}(r, \varphi, t)=-G \int_{0}^{\infty} \Sigma\left(r^{\prime}\right) r^{\prime} \mathrm{d} r^{\prime} \\
& \quad \times \int_{0}^{2 \pi} \frac{\left[z_{m}(r, \varphi, t)-z_{m}\left(r^{\prime}, \varphi^{\prime}, t\right)\right]}{\left[r^{2}+r^{\prime 2}-2 r r^{\prime} \cos \left(\varphi-\varphi^{\prime}\right)+z_{0}^{2}\right]^{\frac{3}{2}}} \mathrm{~d} \varphi^{\prime} .
\end{aligned}
$$

In the above equations $z_{0}$ is the softening parameter introduced to make the integrals regular at $r=r^{\prime} . z_{0}$ can be interpreted as a finite thickness of the disk. The above integral Eq. (3a) can be solved by recasting it into a matrix-eigenvalue problem. On a uniform grid with $N$ radial points the resulting eigenvalue equation for the $m$ th mode takes the form

$\left[\omega_{m}^{2}-2 m \Omega\left(r_{i}\right) \omega_{m}\right] h_{m}\left(r_{i}\right)=\sum_{j=1}^{N} \Lambda_{i j} h_{m}\left(r_{j}\right)$

where

$\Lambda_{i j}=M_{i j}+\delta_{i j}\left(v_{h}^{2}\left(r_{j}\right)+v_{\mathrm{dc}}^{2}\left(r_{j}\right)-m^{2} \Omega\left(r_{j}\right)^{2}\right)$

$M_{i j}=\Delta r G \Sigma\left(r_{j}\right)\left[H\left(r_{i}, r_{j}\right)-K_{m}\left(r_{i}, r_{j}\right)\right] r_{j}$,

and

$v_{\mathrm{dc}}\left(r_{i}\right)=G \int_{0}^{\infty} \Sigma\left(r^{\prime}\right) H\left(r_{i}, r^{\prime}\right) r^{\prime} \mathrm{d} r^{\prime}$.

The above Eq. (5a) can be rewritten in a more compact form:

$\left(\omega_{m}^{2} I+\omega_{m} R+\Lambda\right) h_{m}=0$,

where $I, R$ and $\Lambda$ are the three $N \times N$ real square matrices. $h_{m}$ is the eigenvector corresponding to the eigenvalue $\omega_{m}$.

The matrix elements are $I_{i j}=\delta_{i j}, R_{i j}=-2 m \Omega\left(r_{j}\right) \delta_{i j}$ and $\Lambda_{i j}$ as shown above in Eq. (5b).

\subsection{Asymmetric warp}

The bowl-shaped mode is an axisymmetric bending of the disk and the integral-sign warping mode is anti-symmetric in the azimuthal angle $(\varphi)$. In the linear regime each of the two bending modes behaves much like an independent oscillator in the system, so that we ignore any kind of energy cascading. This is shown to be valid in the last paragraph of Sect. 3. By making a linear combination with proper time dependence of the two bending modes we are able to produce an asymmetric bending mode of the initially assumed axisymmetric disk. Thus according to Eq. (2a) the asymmetric bending mode is described by

$$
\begin{aligned}
\mathcal{Z}_{\text {asym }}(r, \varphi, t) & =A_{\circ} z_{\circ}(r, \varphi, t)+A_{1} z_{1}(r, \varphi, t) \\
& =A_{\circ} \mathfrak{R}\left\{h_{\circ}(r) \mathrm{e}^{\mathrm{i} \omega_{\circ} t}\right\}+A_{1} \mathfrak{R}\left\{h_{1}(r) \mathrm{e}^{\mathrm{i}\left(\omega_{1} t-\varphi\right)}\right\} .
\end{aligned}
$$

So that at any time $t=\tau$ we can analyse the behaviour of the resulting asymmetry. It was proved that the $m=0$ mode and the $m=1$ mode in a disk described above are stable (see Hunter \& Toomre 1969), and their stability was further confirmed by numerical work (see SC88, Sparke 1995). Our numerical work also confirms the earlier result. Thus the resultant asymmetric bending mode can be assumed to be stable and it establishes a classic example of dynamical wave interference pattern in a gravitating medium. The degree of asymmetry in the disk bending depends on the amplitude of the bowl-shaped mode which can be controlled by the free parameter $A_{\circ}$ in the problem. Thus by varying $A_{\circ}$ one can in principle produce a rich class of asymmetries that are seen in the observations. Recent observations show that asymmetric warps are indeed common and they exist in poor environments too (Garcia-Ruiz et al. 2002). This suggests that tidal interactions are not the only process that can produce an asymmetric warp. With our approach, we show how an asymmetric warp can be generated purely from the internal disk dynamics- assuming that these modes are generated due to some mechanism.

\subsection{Disk-halo system}

We have adopted a truncated exponential disk with a $\cos ^{2}$ tapering as introduced by $\mathrm{SC} 88$ :

$$
\begin{aligned}
\Sigma(r) & =\Sigma_{0} \mathrm{e}^{-r / R_{\mathrm{d}}} \quad r \leq r_{\text {trun }} \\
& =\Sigma_{0} \mathrm{e}^{-r / R_{d}} \cos ^{2}\left\{\frac{\pi}{2} \frac{r-r_{\text {trun }}}{r_{\text {out }}-r_{\text {trun }}}\right\} \quad r_{\text {trun }} \leq r \leq r_{\text {out }} \\
& =0 \quad r \geq r_{\text {out }}
\end{aligned}
$$

where $R_{\mathrm{d}}$ is the exponential disk scalelength and $\Sigma$ is the disk surface density. The gradual truncation of the disk avoids spurious modes (SC88). In the calculations (Sect. 3), we have taken $r_{\text {out }}$ and $r_{\text {trun }}$ to be fixed at 6 and 5 disk scalelengths respectively.

The flattened dark matter halo is taken as a screened isothermal halo which gives an asymptotically flat rotation curve (de Zeeuw \& Pfenniger 1988):

$\rho(r, z)=\frac{\rho_{\circ}}{1+\left(r^{2}+\frac{z^{2}}{q^{2}}\right) / R_{\mathrm{c}}^{2}}$

where $R_{\mathrm{c}}$ is the core radius and $q$ is the halo flattening parameter.

The terminal velocity of dark matter halo is given by:

$v_{\mathrm{t}}=4 \pi G \rho_{\mathrm{o}} R_{\mathrm{c}}{ }^{2} q \frac{\sin ^{-1} \sqrt{1-q^{2}}}{\sqrt{1-q^{2}}}$.

\subsection{Numerical details}

Equation (3a) is a linear integral equation which is equivalent to an infinite dimensional eigenvalue Eq. (6). To simplify this 
problem, a galactic disk of finite radius $\left(r_{\text {out }}\right)$ is approximated as a system of $N$ uniformly spaced rings. Then the resulting problem reduces to an $N$-dimensional eigenvalue problem in the eigenvalue $\omega_{m}$ and $N$-dimensional eigenvector $h_{m}$ describing the shape of the bent disk. This requires the solution of an $N \times N$ matrix. The softening parameter $z_{0}$ is introduced to make the self-gravitating integral (Eq. (4b)) regular along the diagonal line of the $N$-dimensional matrix. We have carried out the numerical computation for different values of the matrix dimension $N$. We note that the eigen frequency of the ground state of either mode ( $m=0$ and $m=1$ ) is not very sensitive to the matrix dimension $N$ or the softening parameter $z_{0}$ in the problem. Numerical results show that as the value of $N$ increases or the softening $z_{0}$ decreases, the ground-state eigen-frequency $\omega_{1}$ of the warping mode $(m=1)$ reduces extremely slowly. For example over an augmentation of 200 rings the change in $\omega_{1} \sim 5.0 \%$. For convenience we have taken $N=80$ for our calculations. A similar calculation was done for the case of the bowl-shaped mode $(m=0)$ but it did not show any definite trend unlike the $m=1$ case. But the change in the frequency $\left(\omega_{0}\right)$ occurs only in the third decimal place and its quite arbitrary. The insensitivity of the ground-state mode frequencies ( $\omega_{0}$ and $\left.\omega_{1}\right)$ on the softening is obvious because these are the integral properties of the disk.

However the ground-state mode shape, $h_{m}$, in either case is quasi-sensitive to the softening parameter. A smooth bending of the disk was obtained for typical values of the softening $z_{0} \sim$ inter ring spacing. We note that a large value of the softening reduces the value of the self-gravitating integral, hence it becomes inefficient to make all the rings precess in a synchronized manner, thus any resultant warping is dissolved. On the other hand if $z_{0}=0$ it is obvious that the self-gravitating integral diverges numerically. Since we are not interested in investigating the structure of the either mode in the resolution $\sim$ inter-ring separation, we keep the softening parameter $z_{0} \sim$ inter-ring spacing and this gives us a satisfactory result for the mode shape. The sensitivity of the mode shape on the softening is understandable because a global mode shape depends on the local details of the disk.

For $m=0$, i.e. bowl-shaped mode, the eigenvalue Eq. (6) is linear in $\omega_{0}^{2}$. But for $m=1$, i.e. a warping mode, the problem reduces to a quadratic eigenvalue problem (known as QEP in the literature) in $\omega_{1}$. The standard way to solve the QEP (6) for the $m=1$ mode is to reduce it to a generalized eigenvalue problem (GEP) of the form $A x=\omega_{m} B x$ of twice the matrix dimension $2 \mathrm{~N}$ see Bai et al. (2000). This GEP is commonly called a linearization of the QEP (6). We solve the linearized QEP numerically by using standard technique for diagonalization. For further details about solving QEP the readers are referred to Guo (2004) and Higham \& Kim (2000).

\section{Results}

Recent observations have shown that the galactic disks bend with a variety of shapes starting from symmetric "S" shaped warps to asymmetric warps and also "L" shaped and "U" shaped warps (Sect. 1). We next show that this rich variety of warp shapes observed can be explained naturally right from

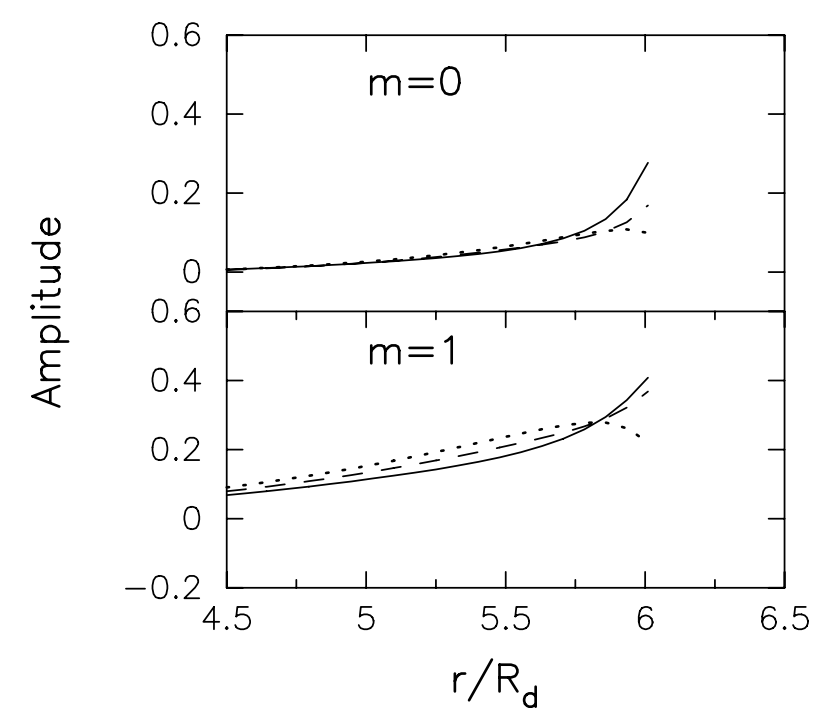

Fig. 1. The ground state mode shapes and their sensitivity w.r.t. disk edge for the two modes $m=0$ and $m=1$. The mode amplitudes are in arbitrary units. The solid, dashed and dotted lines are for $r_{\text {trun }}=5.0$, 5.5 and 5.95 respectively and $r_{\text {out }}=6.0$. Here, the dark matter halo flattening $q=0.7$ and the core radius $R_{\mathrm{c}}=2.0$.

the internal disk dynamics by a superposition of the $m=1$ and $m=0$ modes. The superposition picture proposed and studied here is verified for a few cases in the results from the $N$-body simulations, and there is evidence for an asymmetric warp arising due to a superposition of $m=1$ and $m=0$, or $m=1$ and $m=2$ modes (Revaz \& Pfenniger 2005, personal communication), this requires further study using $N$-body simulations. Since we superpose two modes, the range of values spanned cover the two frequencies $\omega_{0}, \omega_{1}$, the superposition amplitudes $A_{0}, A_{1}$, and the two phases. The unmodulated amplitudes $h_{1}$ and $h_{0}$ (see Eq. (2b)) are free upto a linear multiplication factor. These and the frequencies are obtained from our modal analysis. The ratios of $\omega_{0} / \omega_{1}$ are seen to vary from 8-20 when the flattening of the dark matter halo $(q)$ varies from 0.5 to 0.9 . (See the discussion at the end of this section.) We do not have any a priori physical basis to choose the values of $A_{0}$ and $A_{1}$, these are set by the generation process of the modes - hence we cover a reasonable range for these.

All the numerical calculations are done in units of $G=$ $M_{\mathrm{d}}=R_{\mathrm{d}}=1$ where $M_{\mathrm{d}}$ is the disk mass. In order to show the basic results first, we have fixed the dark matter halo parameters at $R_{\mathrm{c}}=2.0$ (in units of $R_{\mathrm{d}}$ ), $\rho_{0}=0.0117$ (in units of $M_{\mathrm{d}} / R_{\mathrm{d}}^{3}$ ) with a halo flattening parameter $q=0.7$. These give rise to the terminal velocity of the halo as $v_{\mathrm{t}}=0.68$ (in units of $\left.\sqrt{G M_{\mathrm{d}} / R_{\mathrm{d}}}\right)$.

In order to check our numerical calculations with the previous works by SC88 and Sparke (1995) we have reproduced the ground state mode shape for the modes $m=0$ and $m=1$ for some typical disk and halo parameters as can be seen in Fig. 1. The amplitude of these modes are measured with respect to the inner disk plane and these we call as the unmodulated amplitudes. In addition to this, Fig. 1 also shows the sensitivity of the amplitudes of these modes with respect to disk edge. To study the sensitivity we have varied $r_{\text {trun }}$ keeping the disk outer edge 

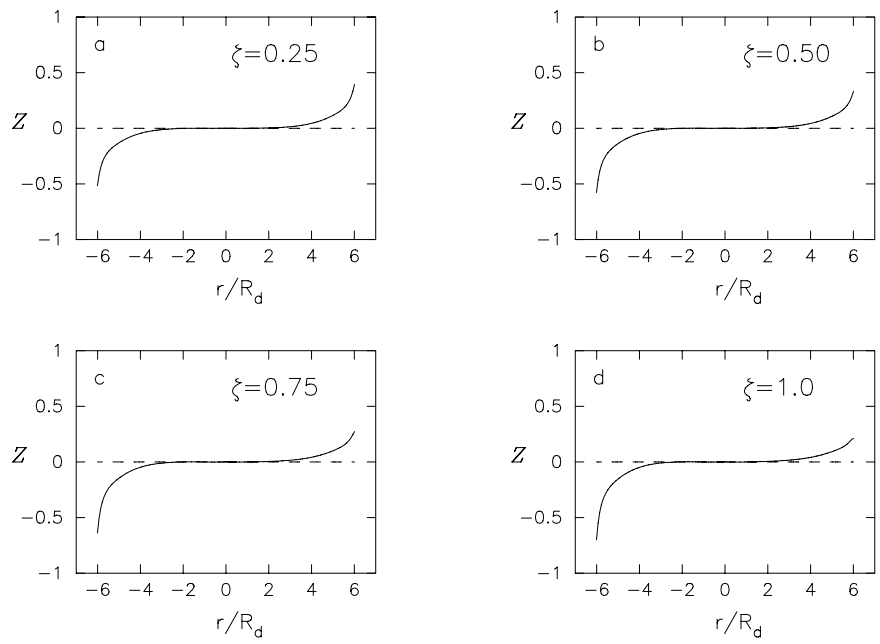

Fig. 2. A plot of the bending amplitude of asymmetric warp versus radius, for various values of the parameter $\zeta=A_{0} / A_{1}$ denoting the ratio of the superposition amplitudes, at an epoch $t=\tau_{\circ} / 8$. As $\zeta$ increases to 1 , the asymmetry becomes prominent. Here, the dark matter halo flattening $q=0.7$ and the core radius $R_{\mathrm{c}}=2.0$.

fixed. The solid, dashed and dotted lines are for $r_{\text {trun }}=5.0$, 5.5 and 5.95 in both the figure panel. The disk density starts deviating from the exponential from $r=r_{\text {trun }}$ and smoothly tapers to zero at $r=r_{\text {out }}$. The dotted curves show the behaviour of the modes when the disk is almost abruptly truncated and in this case too the two modes $m=0$ and $m=1$ behave in the same way. Also note that the amplitudes of $m=0$ modes are slightly less than that of $m=1$ modes. The eigen frequencies corresponding to these ground states are not quite sensitive to the behaviour of disk edge which can be seen from the SC formula for the modified tilt mode (Eq. (21) in SC88). This actually confirms what we get numerically for the $m=1$ mode. However for the $m=0$ modes the eigen frequencies vary from 0.1315 to 0.1296 as $r_{\text {trun }}$ varies from 5.0 to 5.95.

In Fig. 2 we have shown four subplots of an asymmetric warp generated with various values of the controlling parameter $\zeta=A_{0} / A_{1}$ which we will use as an indicator of the relative strength of the bowl-shaped mode. $A_{0}=1.0$ and $A_{1}=1.0$ connotes that the bowl-shaped mode and the ' $S$ ' shaped warping mode both are present with their full unmodulated amplitudes, $h_{m}$ (see Eq. (2b)). These plots are made for an epoch of $\tau_{0} / 8$ as an illustrative case, where $\tau_{0}=2 \pi / \omega_{0}$ is the characteristic time scale in the problem and $\omega_{0}$ is the ground state eigenfrequency of the bowl-shaped mode. As we move from Figs. 2a to 2d, the value of the controlling parameter $\zeta$ increases, that is, the relative strength of the bowl-shaped mode increases. Since in our analysis bowl-shaped mode is the only cause of asymmetry in the bending, we can see from Fig. 2 d, that the warp is most asymmetric i.e. the degree of asymmetry is the highest.

In Fig. 3, we have produced asymmetric warps at different epochs $(t)$ keeping the controlling parameter $\zeta=1.0$. The values of the sampling times $t$ were so chosen that as we move from Figs. 3a to 3d, we see the diverse phenomena of disk warping including symmetric as well as asymmetric ones. This is due to the characteristic oscillation of the disk in a bowlshaped mode. The disk oscillates from "cupped upward" to flat,
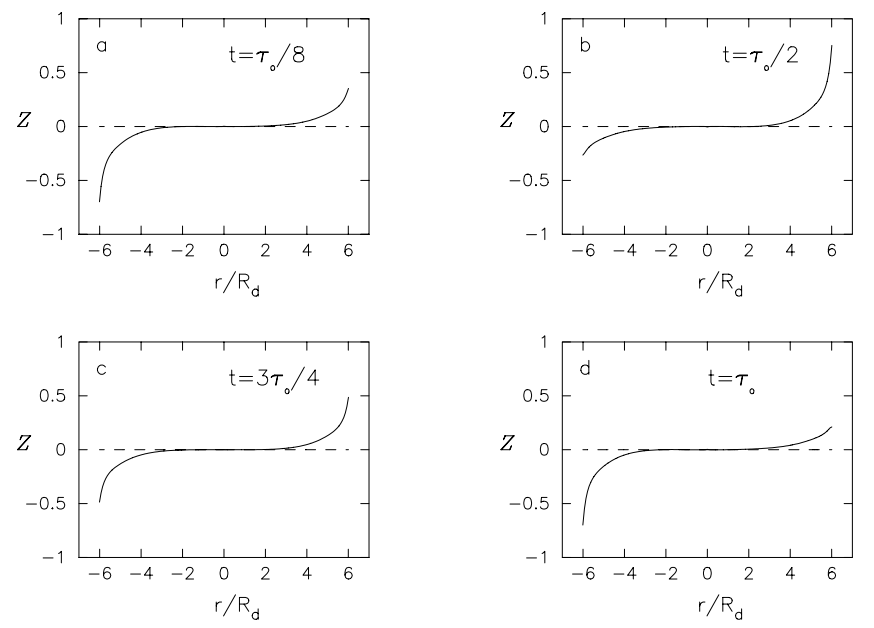

Fig. 3. A plot of the bending amplitude of the asymmetric warp versus radius, at different epochs $t$. As $t$ goes through the cycle of the bowl-shaped mode it generates various kinds of disk warping from an asymmetric to a symmetric and then an asymmetric case. Again, the dark matter halo flattening $q=0.7$ and the core radius $R_{\mathrm{c}}=2.0$.

to "cupped downward" and back again with its typical ground state frequency $\omega_{0}$. During this flexing of the disk it gets a chance to interfere with the S-shaped mode of the disk and this produces a rich class of dynamical asymmetric figures. In a real galaxy the bowl-shaped mode may decay due dissipative effects. Even then the mild asymmetries in disk warping which are seen in observations can be considered as a signature of the bowl-shaped mode.

In the above two figures (Figs. 2-3) we have shown the warping of the disk with respect to the inner unwarped disk plane because in actual observations the warping is quantified based on measurements with respect to the inner disk plane. Next, we define a quantitative measure of the asymmetry seen in a disk galaxy, the asymmetry index, as follows (see also Sanchez-Saavedra et al. 2003):

$\alpha_{\text {asym }}=\frac{\left|\alpha_{\text {right }}-\alpha_{\text {left }}\right|}{\alpha_{\text {right }}+\alpha_{\text {left }}} \quad$ if $\quad \alpha_{\text {right }}^{2}+\alpha_{\text {left }}^{2} \neq 0$

where $\alpha_{\text {right }}$ is the angle between the line joing the centre to the outer most point on the right hand side of the particular warp and the inner disk plane. $\alpha_{\text {left }}$ is defined similarly. Thus $\alpha_{\text {asym }}$ is the normalized or fractional value of the asymmetry. In terms of this definition, the results in Figs. 2 and 3 give an asymmetry of $\alpha_{\text {asym }} \sim 0.2-0.4$. Thus our results for asymmetry agree with the typical range of measured values in Sanchez-Saavedra et al. (2003) and the asymmetries when measured from the figures given in Appendices (A and B) in Schwarzkopf \& Dettmar (2001). For example the value of asymmetry index for the S-shaped asymmetric warp(galaxy name- AM 1134-323(R)) when measured from Fig. 6 in Schwarzkopf \& Dettmar (2001) gives a value 0.33 . This agrees closely with what we get from Fig. 2. Of course true comparisons with observations is a bit tricky because of the free parameters $\left(A_{0}\right.$ and $\left.A_{1}\right)$ involved in our problem. Our basic idea is to show that with this modal superposition approach, one can reproduce various asymmetric warps that are seen in observations. 
Clearly when $\alpha_{\text {right }}=\alpha_{\text {left }}$ the asymmetric index $\alpha_{\text {asym }}$ vanishes. This implies a purely symmetric warping of the disk. Note that Eq. (11) avoids the possibility of $\alpha_{\text {right }}$ and $\alpha_{\text {left }}$ being simultaneosly zero as the case is not physically meaningful. When $\alpha_{\text {right }}=0$ or $\alpha_{\text {left }}=0$ we obtain unity value of the asymmetric index. So $\alpha_{\text {asym }}=1$ (maximum asymmetry) denotes a perfectly "L" shaped warping of the disk. This can be called a one-sided warp. The values of $\alpha_{\text {asym }}$ vary from 0.0 to 1.0 . It is obvious that most of the geometrical shapes of galactic warps seen in observations can be classified using a single parameter $\alpha_{\text {asym }}$. Jimenez-Vicente et al. (1997) used a different set of 3 parameters to describe the shape of warps. However neither their model nor $\alpha_{\text {asym }}$ alone can describe the peculiar shape of the warp of our Galaxy. But $\alpha_{\text {asym }}$ being a single parameter is very useful in depicting the underlying asymmetries in warps. Given the extreme importance of this parameter, we next study the dependence of $\alpha_{\text {asym }}$ on the dark matter halo parameters.

Figure 4 shows the variation of the asymmetry index, $\alpha_{\text {asym }}$, due to the variation in $q$, the flattening parameter of the dark matter halo for a fixed value of the controlling parameter $(\zeta=1)$ and at an epoch $t=\tau_{0} / 8$ as a special case. As the flattening parameter of a halo of fixed mass is varied its central density, $\rho_{0}$, and the core radius, $R_{\mathrm{c}}$ are bound to change. Therefore we need to calculate the $\rho_{0}$ and $R_{\mathrm{c}}$ as functions of $q$. By imposing two constraints: the mass within a thin spheroidal shell(Binney \& Tremaine 1987, p. 54) and the terminal velocity $\left(v_{\mathrm{t}}\right.$, see Eq. (10)) of the halo should be independent of $q$, we obtain $\rho_{0}(q)$ and $R_{\mathrm{c}}(q)$ in terms of their spherical counterparts as (see Narayan et al. 2005):

$\rho_{\circ}(q)=\rho_{\circ}(1) \frac{1}{q}\left(\frac{e}{\sin ^{-1} e}\right)^{3}, \quad R_{\mathrm{c}}(q)=R_{\mathrm{c}}(1)\left(\frac{\sin ^{-1} e}{e}\right)$

where $e=\left(1-q^{2}\right)^{1 / 2}$. As the halo becomes more flattened (smaller $q$ ), the resulting asymmetry $\alpha_{\text {asym }}$ goes down which means that the warp becomes more symmetric. Conversely, as the halo becomes more spherical the asymmetric index $\alpha_{\text {asym }}$ goes up showing that asymmetric warps are more likely to be found in a less oblate dark matter halo. This can be explained as follows. As a constant-mass halo is flattened, the ground state eigenfrequency $\left(\omega_{0}\right)$ of the bowl-shaped mode increases, so that the disk flexes up and down more rapidly. Therefore the characteristic timescale $\left(\tau_{0}\right)$ of the mode decreases and thereby its unmodulated amplitude reduces faster at the measured epoch, this in turn reduces the asymmetry of the warps. While this figure illustrates an important physical point from the model, its direct observational verification is not possible.

In Fig. 5 we plot the variation of the asymmetry index, $\alpha_{\text {asym }}$, with the core radius $R_{\mathrm{c}}$ of the dark matter halo (given in units of $R_{\mathrm{d}}$ ), again for a fixed value of the controlling parameter $(\zeta=1)$ and at an epoch $t=\tau_{0} / 8$ and for a halo flattening of $q=0.75$. In contrast to Fig. 4 , here as $R_{\mathrm{c}}$ increases, the halo mass within a given radius goes up. Figure 5 shows that asymmetry in warps are likely to be more in a halo with a smaller core radius; while as $R_{\mathrm{c}}$ increases, $\alpha_{\text {asym }}$ goes down. This is because as $R_{\mathrm{c}}$ and hence the halo mass increases, the frequency $\omega_{\circ}$ of the bowl-shaped mode is raised, and this results in a smaller intrinsic or unmodulated amplitude of the

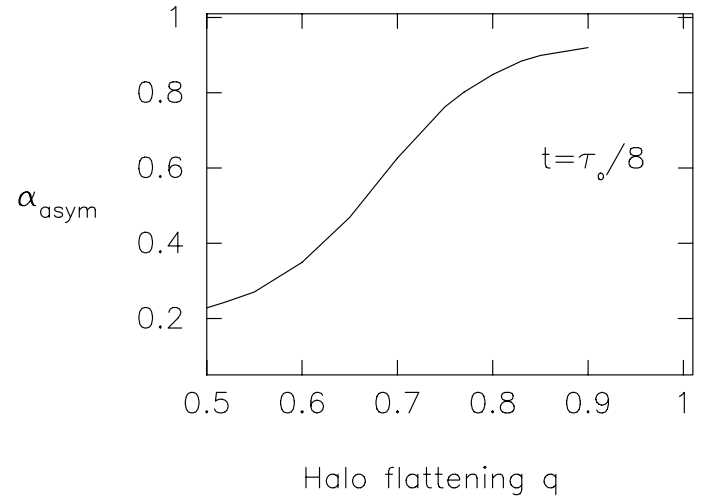

Fig. 4. A plot of the asymmetry index, $\alpha_{\text {asym }}$, versus $q$, the dark matter halo flattening. As the halo becomes more spherical, the asymmetry in warps goes up. Here, the dark matter halo is flattened in such a way as to conserve the ratio of halo mass to disk mass within optical radius of the disk, and the terminal velocity of the halo.

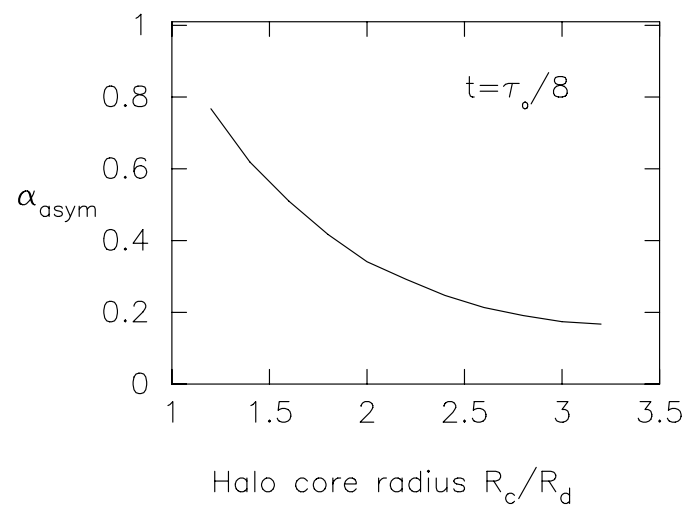

Fig. 5. A plot of the asymmetry index, $\alpha_{\text {asym }}$, versus $R_{\mathrm{c}}$, the dark matter core radius. This shows that as the halo core radius and the halo mass increase, the asymmetry in warps reduces. Here, the dark matter halo flattening is kept constant at $q=0.75$.

bowl-shaped mode. So in absence of an appreciable amplitude of the bowl-shaped mode, the resulting asymmetry in the disk bending also reduces. This result of decreasing asymmetry in warps for higher mass galaxies is in agreement with observations (Castro-Rodriguez et al. 2002), which further supports our model.

The variation of the asymmetry index $\alpha_{\text {asym }}$ on the halo mass can be seen from the Fig. 5. It is explained above that as $R_{\mathrm{c}} / R_{\mathrm{d}}$ increases and hence the halo mass increases the asymmetry index goes down. While as the halo mass decreases the amplitude of warping mode $(m=1)$ starts decreasing and in the limiting case when the dark matter halo is absent $m=1$ mode turns out to be a trivial tilting of the disk. And it is hard to generate $m=0$ mode in the absence of a dark matter halo. Thus in the limiting case when halo mass is tending to zero, the asymmetry index starts falling down to zero and however as we have checked that this happens when $R_{\mathrm{c}} / R_{\mathrm{d}}$ starts becoming a fraction which is not a physically acceptable regime of $R_{\mathrm{c}} / R_{\mathrm{d}}$ for any reasonable disk-halo system known.

So far we have considered a screened isothermal $\left(\rho \propto r^{-2}\right.$ at large radii) dark matter halo, producing a flat rotation curve, for the generation of asymmetric warps. The flaring of $\mathrm{HI}$ in 


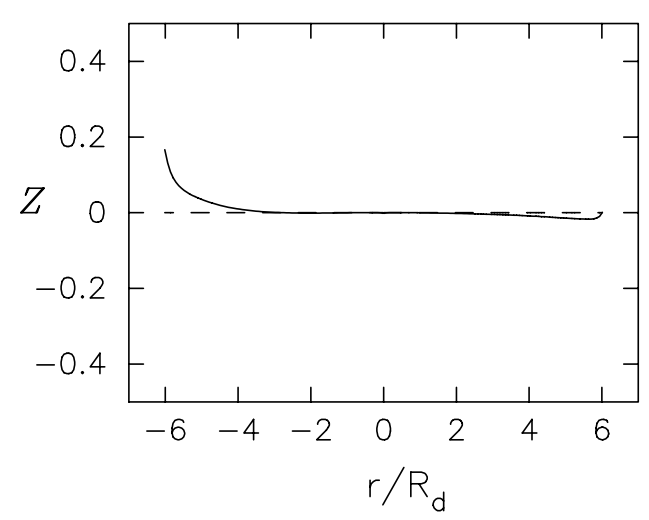

Fig. 6. A plot of bending amplitude versus radius which shows an L-shaped asymmetric warp produced, by setting $A_{0}=0.55$ and $A_{1}=0.20$ at an epoch $t=\tau_{\circ} / 8$. The dark matter halo flattening is kept at $q=0.76$ and $R_{\mathrm{c}}=2.0$.

the outer region of our Galaxy favours a dark matter halo with a steeply falling density profile $\left(\rho \propto r^{-3}\right.$ or $r^{-4}$ at large radii) (Narayan et al. 2005). Note that $\rho \propto r^{-3}$, at large radii, is the popular NFW density profile (Navarro et al. 1996) for the dark matter halo. We have checked that the amplitudes of the two modes ( $m=0$ and $m=1)$ and their ground state eigen frequencies behave in a similar fashion in both cases when $\rho \propto r^{-2}$ and $\rho \propto r^{-3}$ or $r^{-4}$ at large radii. Hence we can say that qualitative behaviour of the asymmetry would remain similar. However we would like to carry a detailed investigation of the asymmetric warp with respect to differnet halo profiles in a future work.

In Fig. 6 we show one of the extreme form of asymmetry of warp shapes, namely an L-shaped mode (see above discussion following Eq. (11)). In principle this can be achieved by reinforcing the net amplitude of bending resulting from the superposition of the two modes to zero on one side of the disk. We get an approximately L-shaped mode for $A_{0}=0.55$ and $A_{1}=0.20$ for the usual input values of $q=0.76$ and $R_{\mathrm{c}}=2.0$. Clearly the root of such an extreme form of asymmetric warp lies in the fine balancing between the two modes in the process of superposition. Nearly $5 \%$ of the asymmetric warps observed have this structure (Sanchez-Saavedra et al. 2003), which can be explained by our approach.

Another form of deviation from an S-shaped distribution is a U-shaped warp, which comes about naturally when the amplitude $A_{0}$ of the bowl-shaped ( $m=0$ ) mode is enhanced w.r.t. the amplitude $A_{1}$ of the warping ( $m=1$ ) mode (see Fig. 7). Nearly $7 \%$ of the asymmetric warps observed have this structure (Sanchez-Saavedra et al. 2003). A little juggling of $A_{1}$ and $A_{0}$ values is required to obtain the $\mathrm{L}$-shaped and the $\mathrm{U}$-shaped modes, unlike the case of the other asymmetric warps. This perhaps explains their lower observed frequency.

Thus our approach can naturally produce the variety of asymmetry seen in disk galaxies, including the peculiar Lshaped and U-shaped warps. The resulting values of asymmetry (Figs. 4-5) agree with the range of observed values (Sanchez-Saavedra et al. 2003 and Schwarzkopf \& Dettmar 2001).

In order to compare the resulting asymmetry with the observed values, it is useful to obtain the true value of $\tau_{0}$. We have

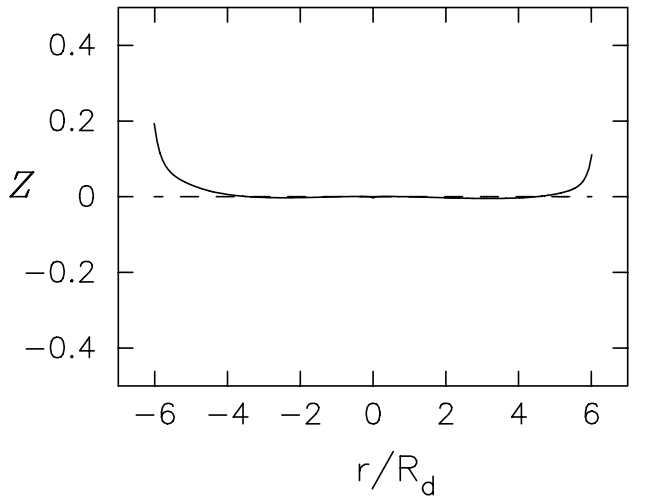

Fig. 7. A plot of bending amplitude versus radius which shows an U-shaped asymmetric warp produced by setting $A_{0}=1.0$ and $A_{1}=0.1$ at an epoch $t=\tau_{\circ} / 8$. The dark matter halo flattening is kept at $q=0.76$ and $R_{\mathrm{c}}=2.0$.

checked that this lies in the range of $\sim 0.5-1.0 \mathrm{Gyr}$ and $\tau_{1}$ lies in the range of $\sim 6-12 \mathrm{Gyr}$, for the realistic galaxy models with a range of $R_{\mathrm{d}} \sim 2-3 \mathrm{kpc}, M_{\mathrm{D}}$, the disk mass $\sim 2-6 \times 10^{10} M_{\odot}$, $R_{\mathrm{c}} \sim 2 R_{\mathrm{d}}, q=0.7$ and the ratio of the halo mass to the disk mass is $\sim 1.4$ within the outer radius $\left(\sim 6 R_{\mathrm{d}}\right)$. The ratio of the frequencies $\omega_{0} / \omega_{1}$ is $\sim 10$, note that this value is obtained for $q=0.7$. For other values of $q$ the ratio $\omega_{0} / \omega_{1}$ varies. Numerical calculations yield a range of values varying from 8-20 for $q$ varying from 0.5 to 0.9 respectively. In the absence of any dissipation, the superposition of the two modes will show a cyclical variation and hence the above values represent the typical asymmetries seen. Even if there is dissipation, so long as there is a regeneration of the modes (see Sect. 1), then such asymmetric warps can recur and hence can be seen effectively over much larger timescales. We note that since the natural frequencies of the two modes are so different, we are justified in treating the problem as a simple linear superposition rather than having to take account of the mode coupling.

\section{Conclusions}

We propose and study the origin of the asymmetric warps in spiral galaxies as arising due to the superposition of the standard S-shaped warps ( $m=1$ mode) and a bowl-shaped warp ( $m=0$ mode). This is a simple but general model for generating asymmetric warps that is independent of whether these modes arise due to tidal interaction, or gas accretion, or bending instabilities, or any other perturber. We do a modal analysis of a disk embedded in a dark matter halo, and obtain the solutions for these two modes which are then linearly superposed. In the characteristic oscillation of the $m=0$ mode, the disk oscillates from "cupped upwards" to flat, to "cupped downwards" and back again. During this flexing of the disk, it gets a chance to interefere with the S-shaped $(m=1)$ mode of the disk, and this produces a rich class of dynamical, asymmetric warps. The results obtained naturally explain the wide variety of asymmetric warps observed in spiral galaxies, including the peculiar Lshaped and U-shaped warps.

Acknowledgements. We are happy to acknowledge useful e-mail correspondence on the $m=0$ mode with Linda Sparke. We are grateful to 
Yves Revaz and Daniel Pfenniger for analyzing some of their $N$-body simulations data to check that there is evidence for the superposition idea proposed here. We also thank them and Françoise Combes, and Martin Lopez-Corredoira for critical comments on the manuscript. We thank the referee Eduardo Battaner for constructive comments on the paper. The numerical package LAPACK (see www. netlib.org) was used for solving the matrix diagonalization. K.S. thanks the CSIRUGC, India for a Senior research fellowship.

\section{References}

Bai, Z., Demmel, J., Dongarra, J., Ruhe, A., \& van der Vorst, H. 2000, ed., Templates for the Solution of Algebraic Eigenvalue Problems: A Practical Guide (Philadelphia: SIAM)

Battaner, E., Florido, E., \& Sanchez-Saavedra, M. L. 1990, A\&A, 236, 1

Binney, J., Jiang, I.-G., \& Dutta, S. 1998, MNRAS, 297, 1237

Binney, J., \& Tremaine, S. 1987, Galactic Dynamics (Princeton: Princeton Univ. Press)

Bosma, A. 1978, Ph.D. Thesis, University of Groningen

Burton, W. B. 1988, in Galactic and Extragalactic Radio Astronomy, ed. G.L. Verschuur, \& K. I. Kellermann (Berlin: Springer), 295

Castro-Rodriguez, N., Lopez-Corredoira, M., Sanchez-Saavedra, M. L., \& Battaner, E. 2002, A\&A, 391, 519

de Zeeuw, T., \& Pfenniger, D. 1988, MNRAS, 235, 949
Dubinski, J., \& Kuijken, K. 1995, ApJ, 442, 492

Garcia-Ruiz, I., Sancisi, R., \& Kuijken, K. 2002, A\&A, 394, 769

Guo, C.-H. 2004, Linear Algebra Appl., 385, 391

Higham, N. J., \& Kim, H.-M. 2000, IMA J. Numer. Anal., 20, 499

Hunter, C., \& Toomre, A. 1969, ApJ, 155,747

Jimenez-Vicente, J., Porcel, C., Sanchez-Saavedra, M. L., \& Battaner, E. 1997, Ap\&SS, 253, 225

Lopez-Corredoira, M., Betancort-Rijo, J., \& Beckman, J. E. 2002, A\&A, 386, 169

Narayan, C. A., Saha, K., \& Jog, C. J. 2005, A\&A, 440, 523

Navarro, J. F., Frenk, C. S., \& White, S. D. M. 1996, ApJ, 462, 563

Nelson, R. W., \& Tremaine, S. 1995, MNRAS, 275, 897

Reshetnikov, V., \& Combes, F. 1998, A\&A, 337, 9

Reshetnikov, V., \& Combes, F. 1999, A\&A S, 138, 101

Revaz, Y., \& Pfenniger, D. 2004, A\&A, 425, 67

Sanchez-Saavedra, M. L., Battaner, E., \& Florido, E. 1990, MNRAS, 246, 458

Sanchez-Saavedra, M. L., Battaner, E., Guijarro, A., LopezCorredoira, M., \& Castro-Rodriguez, N. 2003, A\&A, 399, 457

Sancisi, R. 1976, A\&A, 53, 159

Schwarzkopf, U., \& Dettmar, R.-J. 2001, A\&A, 373, 402

Sellwood, J. 1996, ApJ, 473, 733

Semelin, B., \& Combes, F. 2005, A\&A, 441, 55

Sparke, L. S. 1995, ApJ, 439, 42

Sparke, L. S., \& Casertano, S. 1988, MNRAS, 234, 873 (SC88)

van der Kruit, P., \& Searle, L. 1981, A\&A, 95, 105 